\title{
A Lateral Stability Monitor for Two Wheeled Tractor - Trailer System
}

\author{
Divaker Chelladurai
}

\begin{abstract}
The tractor and two wheeled trailer combination being the most popular load carrying vehicle of the Indian farms, is often susceptible to serious accidents due to lateral roll over. A simple PIC based lateral stability monitor was developed from basic principles for the tractor trailer combo. The system uses a commercial tilt sensor to detect the roll angle, a potentio-metric sensor to sense the turning radius and a hall effect sensor to measure the speed of travel. The control code calculates the lateral stability ratio and compares it to the stability limits. The code cautions the operator visually and audibly to take evasive action well before instability occurs. The monitor was tested in the field under various sloping terrains, turning speeds and payloads, and was found to function satisfactorily.
\end{abstract}

Keywords-Lateral stability, PIC based monitor, tilt sensor, tractor-trailer system,

\section{INTRODUCTION}

$\mathrm{W}$ ikipedia defines dynamic stability control in vehicles stability by detecting and reducing loss of traction. When the control system senses a loss of steering control, it applies brakes to steer the vehicle properly. A road vehicle that traverses a relatively smooth surface is not the same as a field vehicle such as a tractor. Although stability control of off-road vehicles are much needed, not much has been done for tractors, that too for the tractor trailer combo, where many accidents occur in India due to linear and lateral instability. ROF (Roll over frame) a safety accessory, is also not made mandatory in India for tractors.

The Indian Council of Agricultural Research [1] has reported that out of the total farm accidents reported during the last one year, 30 per cent was due to farm machines out of which about 31 per cent of accidents were due to tractors and their equipment. A considerable part of these accidents are due to the rolling over of tractors. Tractors are work horses of the Indian farm, but not given due safety provisions in these lines. Moreover, the tractor trailers are usually manufactured in India by small manufacturers unmindful of stability concerns. Though sophisticated control algorithms, as reviewed next are available for road vehicles, tractors may require a much simpler but unique stability controls since they are relatively slow and traverse farm terrain, many times towing loaded trailers.

Collins and Wong [2] studied the linear stability of

Revised Manuscript Received on December 15, 2019.

Divaker Chelladurai*, School of Agricultural and Processing Sciences, Kalasalingam Academy of Research and Education, Krishnankoil, Tamil Nadu, India. Email:divaker@gmail.com as a computerized technology that improves a vehicle's

* Correspondence Author

articulated vehicles through observing the effects of trailer parameters and tire pressure on the stability of towing a typical car-trailer combo. They indicated that the hitch loading, trailer length, mass and moment of inertia, and variations in the tire pressures influences trailer towing stability. Hac et. al [3] investigated the dynamic stability of an articulated vehicle in the yaw plane through a linear analytical model. Vempaty and He [4] analyzed some active-control schemes, such as active trailer steering control, active trailer braking and model reference adaptive controller through a comparative study of these control strategies. Xie [5] used a simple solid modeling program to compute the inertial properties and thereon the dynamic behavior of the tractor-trailer system. Zhou and Zhang [6] proposed a rollover prevention control method based on real-time calculation of rollover index. Comparing the rollover index with a preset threshold, differential braking was applied to corresponding wheels. Results of simulations showed promising rollover prevention control on high speed curve driving.

This work is about developing a stability monitoring/ warning system that does a two fold job of contending the static lateral stability of the tractor-trailer system by measuring the roll angle; and the dynamic stability of tractor-trailer system, while it executes a turn by measuring the wheel angle and angular velocity. The monitor combines these two parameters in real time into a single stability ratio and compares it with the stability limit to invoke an audible/ visible alarm to the operator well ahead of exceeding the limits.

\section{THEORY OF LATERAL STABILITY OF TWO WHEELED TRACTOR - TRAILER SYSTEM}

The lateral stability of the tractor-trailer system has been derived based on the rollover theory developed by Coombes [7]. Fig. 1 illustrates the rear and side views of the trailer which is about to roll-over laterally. The weight of the trailer along with the payload and the centrifugal force are the two main forces considered, while the trailer is traversing a laterally sloping curve. When the resultant force of all the forces acting on the trailer reaches the boundary of the stability plane, the stability limit is reached, as long as the wheels are not sliding along the contact surface. The stability plane for the trailer attached to the drag bracket on the tractor is that of an inclined triangular plane 'BED', whose apex is the hitch (drag) point on the tractor and the base is the line connecting the ground contact points of the two wheeled trailer wheels. 


\section{A Lateral Stability Monitor for Two Wheeled Tractor - Trailer System}

The sides of this triangle are the lines between the base and rotate about ground contact points of the rear

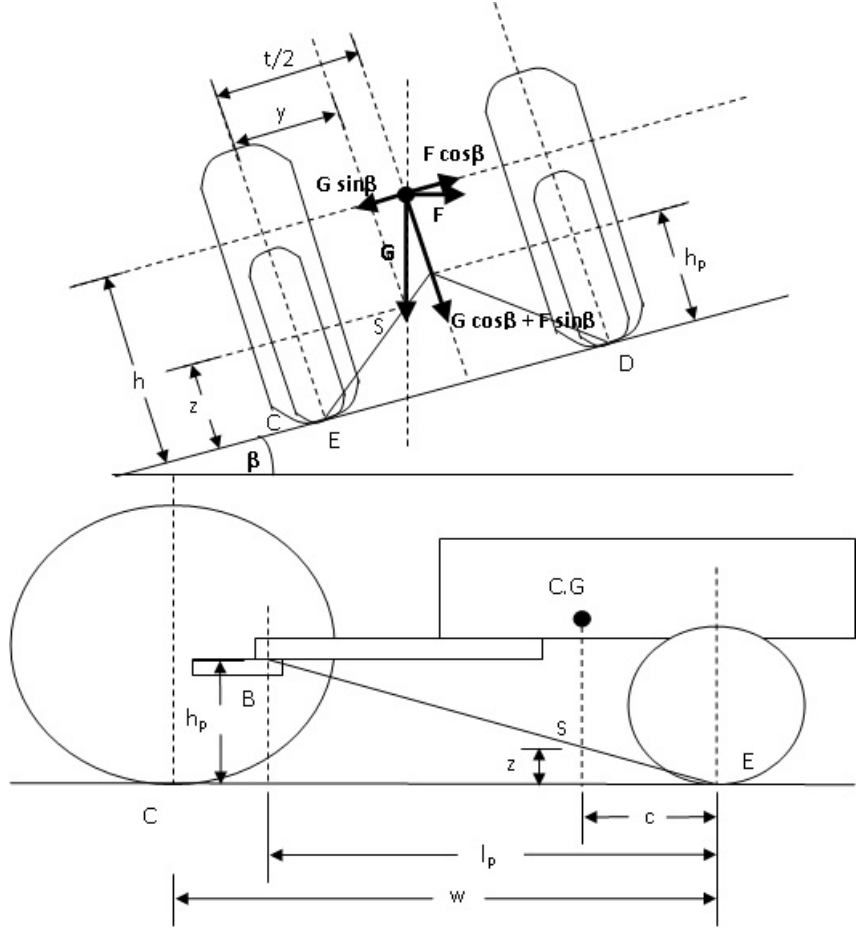

Fig. 1 Two wheeled tractor trailer system

wheel $\mathrm{E}$ and hitch pin $\mathrm{B}$ on the tractor. The force of support at the stability plane's third corner, i.e. the ground contact point of outer trailer wheel D, will go to zero. The resultant force, $\mathrm{G}$ (due to the trailer weight with payload) will be about to pass outside the stability plane intersecting the boundary line EB at $\mathrm{S}$, with $\mathrm{F}$ being the centrifugal force due to turning. Considering rotation about line EB for equilibrium at the point of instability and taking moments about $\mathrm{S}$,

$$
\frac{(\mathrm{G} \sin \beta-\mathrm{F} \cos \beta)}{(\mathrm{G} \cos \beta+\mathrm{F} \sin \beta)}=\frac{(1 / 2 \mathrm{t}-y)}{(\mathrm{h}-\mathrm{z})}
$$

But by symmetry,

$$
\frac{\mathrm{y}}{t / 2}=\frac{\mathrm{c}}{\mathrm{l}_{\mathrm{p}}}=\frac{z}{\mathrm{~h}_{\mathrm{p}}}
$$

Substituting,

$$
\frac{(\mathrm{G} \sin \beta-\mathrm{F} \cos \beta)}{(\mathrm{G} \cos \beta+\mathrm{F} \sin \beta)}=\frac{1 / 2 \mathrm{t}-\left(\frac{1 / 2 \mathrm{tc}}{\mathrm{l}_{\mathrm{p}}}\right)}{\mathrm{h}-\left(\frac{\mathrm{ch}_{\mathrm{p}}}{\mathrm{l}_{\mathrm{p}}}\right)}=\frac{1 / 2 \mathrm{t}\left(\mathrm{l}_{\mathrm{p}}-c\right)}{\mathrm{h}\left(\mathrm{l}_{\mathrm{p}}-\frac{\mathrm{ch}}{\mathrm{h}}\right)}
$$

LHS of (3) is the lateral stability ratio, which depends on the weight, the centrifugal force and the slope (roll) angle of the trailer. RHS is the lateral stability limit which will depend on ' $t$ ', the trailer's tread width, ' $h$ ', its centre of gravity height and ' $h p$ ', trailer hitch height. If there is no centrifugal force involved due to turning on the slope, (3) becomes

$$
\tan \beta=\frac{1 / 2 \mathrm{t}\left(\mathrm{l}_{\mathrm{p}}-c\right)}{\mathrm{h}\left(1_{\mathrm{p}}-\frac{\mathrm{ch}_{\mathrm{p}}}{\mathrm{h}}\right)}
$$

The lateral stability of trailer as from (3) can be increased by any one of the following: the hitch (pivot) point being the apex. The trailer will tend to

- increase ' $t$ ' i.e. widen trailer's tread width;

- decrease ' $h$ ' i.e. lower trailer's centre of gravity by proper payload stacking;

- increase 'hp' i.e. raise trailer hitch point (which may not be feasible)

The first stage of lateral roll-over of the trailer starts at the slope angle $(\beta)$, when all weight is transferred out of the outer (upper) wheel of the trailer axle. However, after the point of travel limit of the pivot is reached, the vehicle body will continue to roll around line EB. It rolls about the line joining the base of the lower (inner right) tire of the tractor and the lower (inner) wheel of the trailer (points E and C in Fig.1). This is the slope angle $(\gamma)$ when the second stage of stability occurs and both the tractor and trailer starts to roll over as a single unit. The instability angle of second stage can be written as

$$
\frac{(\mathrm{G} \sin \gamma-\mathrm{F} \cos \gamma)}{(\mathrm{G} \cos \gamma+\mathrm{F} \sin \gamma)}=\frac{1 / 2 \mathrm{t}}{\mathrm{h}}
$$

And in the absence of the centrifugal force, it is simply

$$
\tan \gamma=(1 / 2 t / h)
$$

\section{CONSTRUCTION AND WORKING OF THE STABILITY MONITORREVIEW CRITERIA}

The stability monitor was built around a commercially available digital tilt sensor comprising of a 3 Axis Linear MEMS Accelerometer and a microcontroller module to implement the stability control algorithm. The ARN- TS2D digital tilt sensor (Made by AERON systems, 502, Mitrangan, Baner Road, Baner, Pune - 411045, MH, India http://www.aeronsystems.com) gives accurate data about roll and pitch axis in serial (RS232) format.

Output is available at continuous rate from the sensor which is using MEMS accelerometers and embedded processing to give accurate information on roll and pitch. These capacitive accelerometers are also less susceptible to noise and temperature variation, dissipate less power, and has large bandwidths due to internal feedback circuitry. TheARN- TS2D tilt sensor is factory calibrated requiring no on- site calibration. Its measurement range is \pm 60 (pitch) and \pm 180 (roll) deg at a resolution of $0.01 \mathrm{deg}$. and an accuracy is $\pm 0.1 \mathrm{deg}$. The sensor is powered by $+12 \mathrm{VDC}$ at $50 \mathrm{~mA}$ with interface options of RS232 ASCII, RS485 ASCII MODBUS.

On RS232 and RS485 serial interface, the sensor gives angles in form of a string through transmit pin TX of connector. This data is composed of ASCII characters and the packet is of the format,

$\mathrm{R}: \mathrm{SXXX} . \mathrm{XX}<$ space $>\mathrm{P}: \mathrm{SXXX}$.XX\r\n corresponding to,

\begin{tabular}{|l|l|l|}
\hline $\begin{array}{l}\text { String/Character } \\
\text { Name }\end{array}$ & Description & Range \\
\hline R: & Roll header & - \\
\hline P: & Pitch header & - \\
\hline S & Sign & '+' or '-' \\
\hline XXX.XX & Angle & Range as per device \\
\hline$\backslash \mathrm{r}$ & Carriage & - \\
\hline $\mathrm{n}$ & Line feed & - \\
\hline
\end{tabular}




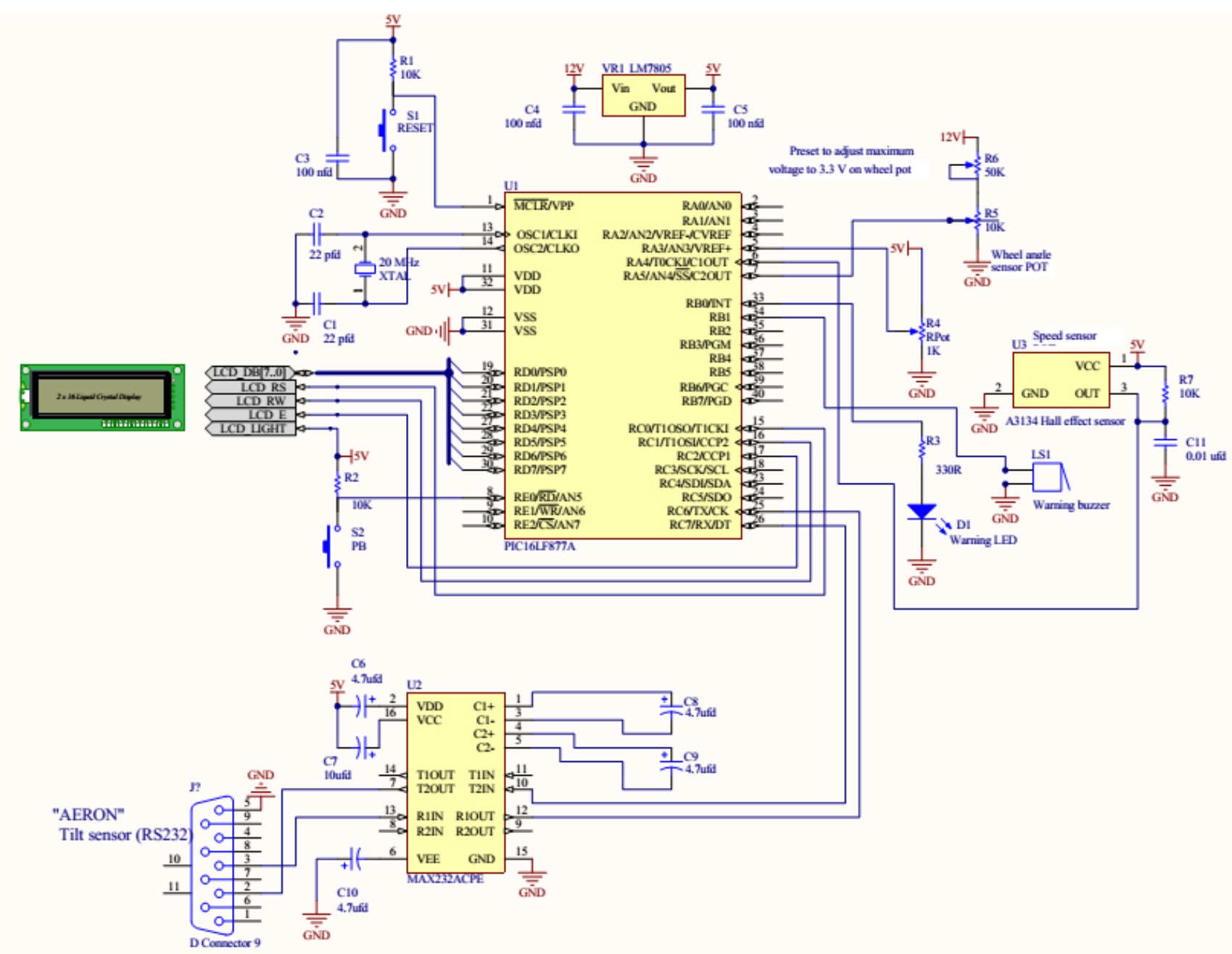

Fig. 2 PIC based stability monitor

\section{THE CONTROLLER}

A 16F877A PIC microcontroller (Fig. 2) was used to develop the stability monitor. The digital tilt sensor is connected to the microcontroller's USART port through a standard MAX232 serial interface IC for level converting of the serial data obtained from the sensor and a DB connector. A preassembled prototype board 'OLIMEX ${ }^{\circledR}$ ' was used for building the controller hardware. The tilt sensor's output is in the form R:+023.44 P:+034.23 $\backslash \mathrm{r} \backslash \mathrm{n}$ as already explained. There are 21 ASCII bytes coming out of the sensor at an output freq of $1.85 \mathrm{hz}$, meaning that this stream is available every $0.54 \mathrm{sec}$.

The roll angle is in the 5th to 9th indices of the array For instance, 23.44 is the roll angle in the given example. This is segregated out and then converted to decimal information. These decimal digits in each place are then combined to read the roll data completely. Hence the roll angle of the trailer is obtained by the microcontroller continuously every $0.54 \mathrm{~s}$.

The front wheel angle of the tractor is also assessed by a simple potentiometric angle sensor. The data obtained from this sensor is used to compute the instantaneous turning radius as well the angular velocity of turning of the tractor - trailer outfit at specific intervals of time. A 45 teeth nylon gear is so mounted on the kingpin axis of the left front wheel of the tractor, which is in constant mesh with a 15 teeth nylon gear mounted on the axis of a linear $10 \mathrm{~K}$ industry grade potentiometer. The potentiometer in turn is mounted appropriately on a plate which is secured to the tractor's chassis. With such an arrangement, the wheel angle which is normally in the range of $50 \mathrm{deg}$ on either side of straight movement is converted to 150 degrees on either side of the potentiometer's mid position. This sums up to a total angular displacement of the POT to $300 \mathrm{deg}$ which is the maximum range of resistance change. A $50 \mathrm{~K}$ preset resistance is connected in series with the wheel angle sensing potentiometer and these in turn across the tractor's $12 \mathrm{~V}$ battery. An approximate adjustment of $26 \mathrm{~K}$ on the preset would provide $3.3 \mathrm{~V}$ maximum, which is the reference voltage set for using the PIC microcontroller's ADC ports. When the analog output is tapped out between the moving contact of the wheel angle POT and the ground, this arrangement outputs $3.3 \mathrm{~V}$ at extreme right turn of the tractor; $3.3 / 2=1.65 \mathrm{~V}$ when the tractor goes straight and $0 \mathrm{~V}$ at extreme left turn of tractor. This analog voltage corresponding to the wheel angle is fed into the AN4th ADC input of the PIC microcontroller (Fig. 2).

A hall effect sensor A3134 ('Allegro ${ }^{\circledR}$ ') was used to configure a wheel speed sensor. The sensor along with the needed resistor circuit was mounted inside a PVC tube which in turn was provided with some mounting plates for fixing

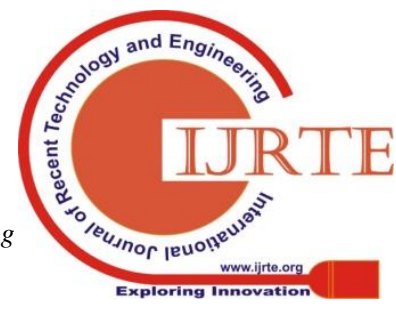




\section{A Lateral Stability Monitor for Two Wheeled Tractor - Trailer System}

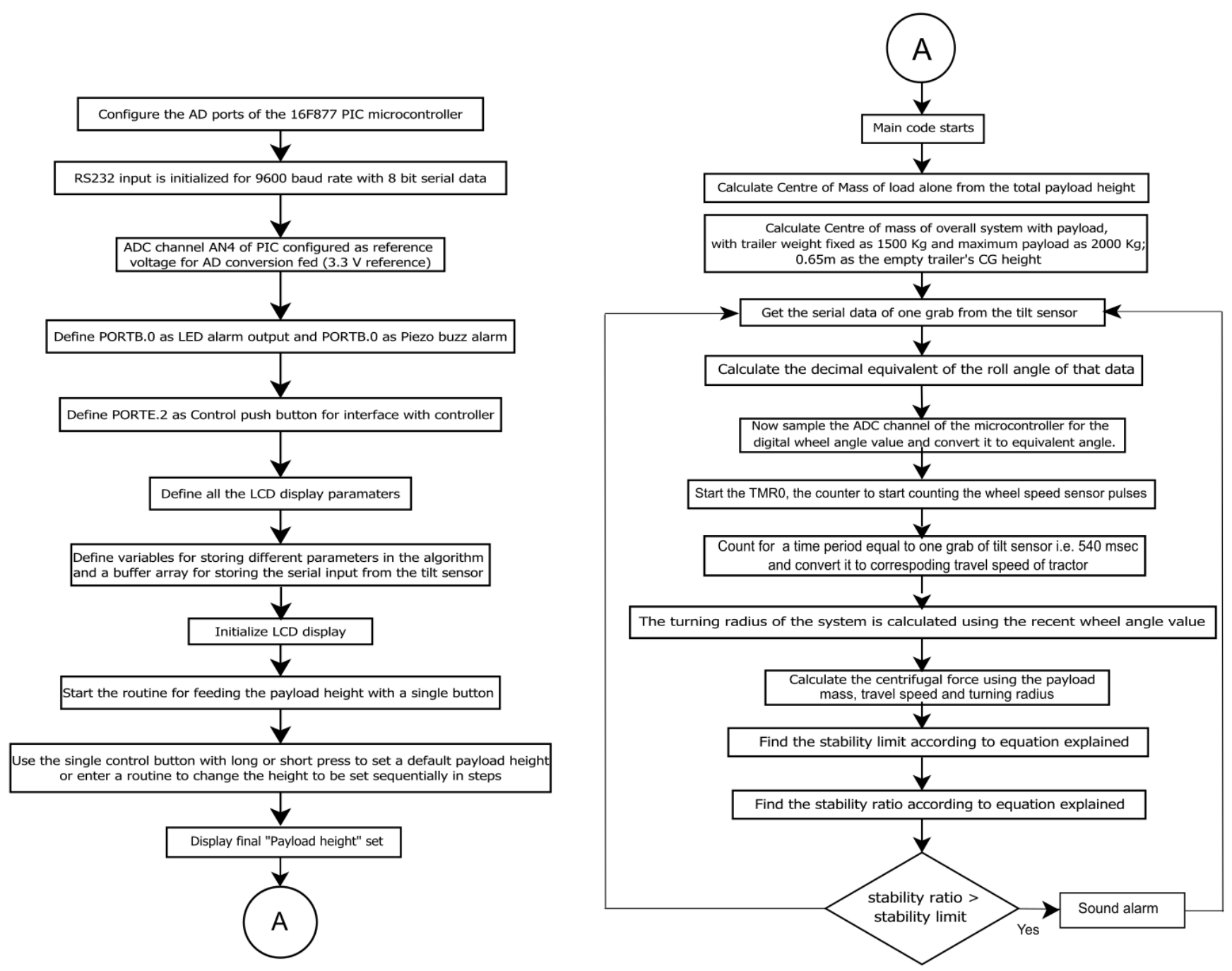

Fig. 3. Program flow

It near the front wheel for speed detection. One of the front wheel hubs was provided with four buttons of permanent magnets of $15 \mathrm{~mm}$ dia on its outer periphery at equi-spacing. The magnets were simply epoxied to the hub in place. The sensor was mounted on the king pin housing such that it directly faces the permanent magnets. The clearance between the sensor and the magnets was adjusted to $5 \mathrm{~mm}$ for maximum sensitivity. When the tractor moves and the front wheel rotates, the sensor detects the magnets one by one and sends out a switched signal. Each revolution of the front wheel then provides 4 pulses. The front wheel's effective road diameter being $0.7 \mathrm{~m}$, the distance travel per revolution would be $\pi(0.7)=2.2 \mathrm{~m}$. Four pulses per revolution of wheel would mean 4 pulses per $2.2 \mathrm{~m}=0.54 \mathrm{~m}$ travel per pulse. Since there is a delay of $0.54 \mathrm{~s}$ between two grabs of tilt, which is also the time available for counting the speed sensor input, the counter would be incremented by the no of pulses that arrive during that time. i.e. (say) $\mathrm{N}$ counts would have been made in $0.54 \mathrm{~s}=1.85 \mathrm{~N}$ counts per second. Again since each pulse count implies $0.54 \mathrm{~m}$ forward travel,

Linear speed of the tractor $=(0.54 \mathrm{~m}$ count -1$) *(1.85 \mathrm{~N}$ counts s-1) $=\mathrm{N}$ ms-1. This implies that the counts accumulated in each sampling cycle is the linear speed in $\mathrm{ms}-1$. Once the linear speed (v) and the turning radius (r) are available, the controller calculates the angular velocity as $\mathrm{v} / \mathrm{r}$ and the centrifugal force as mv2/r. Each time as explained already, the lateral stability ratio, as from the weight, the centrifugal force and the slope (roll) angle of the trailer is calculated and compared against the lateral stability limit which depends on the trailer's tread width, its centre of gravity height and the trailer hitch height. If the limit is exceeded, the alarm buzzer is sounded and warning LED flashed. A safety overhead of 15 per cent was added to the stability ratio calculated so that the alarm is sounded well in advance of the really hazardous stability limit.

The PIC outputs to a LED serving as the stability limit alarm indicator as well to a Piezo sound alarm. It also inputs a push button input, serving as the input device for interacting with the PIC. The PIC is also connected to a $16 \times 2 \mathrm{LCD}$ display module serving to display values of stability criteria and limit at regular intervals, as well for inputting the relevant data such as payload weight and payload height at the initiation of the code. The PIC was programmed by a commercial PIC simulator IDE and hardware. The basic algorithm of detecting the stability limit as explained earlier was built into the code (Fig. 3) so that the stability module looks for any lateral stability concern based on undue tilt of system or excessive centrifugal force and switches on the alarms.

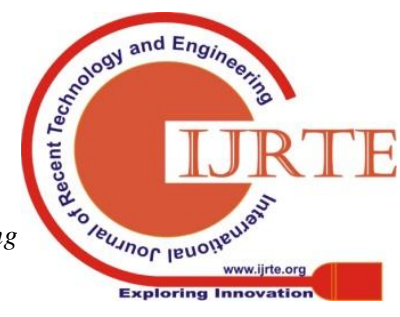




\section{TESTING OF THE MONITOR}

The monitor was field tested with various payloads as well at different turning speeds and terrain slopes. A special third wheel was mounted on to one side of the tractor chassis to test the tractor and trailer without the fear of overturning. The alarms indicated the stability limit quite efficiently for the driver to be cautious. The implemented system is rudimentarily simple and can be extended to include a throttle shut-off control in future. The monitor was found to satisfactorily caution the operator with visual and audio alarms well before the onset of lateral instability membership.

\section{CONCLUSION}

A microcontroller based lateral stability monitor was developed systematically after a theoretical study of the stability dynamics concerning a two wheeled tractor-trailer system. The system dynamics indicated a stability limit equation which was implemented into the controller coding. The tractor's turning speed and radius contended the dynamic lateral stability and the lateral slope and location of centre of gravity, its static stability. Appropriate instrumentation was used to assess these parameters on-the-go and the controller did the job of alerting the operator audibly well ahead, when there is a stability concern. The electronic monitor was tested on live run using a third wheel mounted on the tractor to prevent any possible testing accidents. The trailer payload, speed, turning radius and side slope of terrain were varied to do the test. It was able to caution the operator adequately before any impending stability mishap.

\section{ACKNOWLEDGMENT}

The support offered by the Agricultural Engineering College and Research Institute, Tamil Nadu Agricultural University, Coimbatore, India is acknowledged with gratitude..

\section{REFERENCES}

1. aicrp.icar.gov.in, 'Survey of accidents in Indian Agriculture", 2019. [Online]. Available: https://aicrp.icar.gov.in/esa/survey-of-accidents -in-indian-agriculture/[Accessed:9-Jun- 2019].

2. R. L. Collins and J. P. Wong. "Stability of Car Trailer Systems With Special Regard to Trailer Design”, J. Dyn. Sys.. Meas. Control. 96(2), pp. 236-243, 2010.

3. A. Hac, D. Fulk and H. Che. "Stability and Control Considerations of Vehicle-Trailer Combination", SAE technical paper series, 2008-01-122, 2008 World Congress, Detroit, Michigan, April 14-17, 2008.

4. S. Vempaty and Y. He. "A Review of Car-Trailer Lateral Stability Control Approaches", SAE Technical Paper 2017, 1580, 2017, doi: 10.4271/2017-01-1580.

5. L. Xie. "Simulation of tractor-trailer system stability", Retrospective Theses and Dissertations. 16351. https://lib.dr.iastate.edu/rtd/16351, 1984.

6. S. Zhou and S. Zhang. "Study on Tractor Semi-Trailer Roll Stability Control", The Open Mechanical Engineering Journal, 2014, 8, pp. 238-242.

7. G. B. Coombes. "Slope stability of Tractors". Farm Machine Design Engineering, September 1968, pp.18-33.

\section{AUTHORS PROFILE}

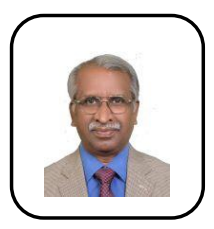

Dr. Divaker Durairaj Chelladurai having a $\mathrm{Ph} . \mathrm{D}$ in Agriicultural Engineering is a former Professor of Farm Machinery and Dean of Agricultural Engineering College and Research Institute, Tamil Nadu Agricultural University, Coimbatore, India. He has a professional experience of 36 years. His research Interests are in the Design of field machinery; Soil tool modeling, Application of Electronics and Computers in Agriculture, Electrostatics for pesticide spraying and Precision Farming Applications. He has implemented number of research projects from DST, UGC and CSRI of India. He had trainings in NIRAE, Tsukuba, Japan and FABE, Ohio State University, USA. He has guided more than 15 students into their $\mathrm{PhD}$ and MTech degrees in Ag Engineering and has published over 30 research papers in Peer reviewed International scientific journals and two books/ book chapters. $\mathrm{He}$ is also a life member of the Indian Society of Agricultural Engineers. He has been an expert member of policy making and research review committees for ICAR at National level.

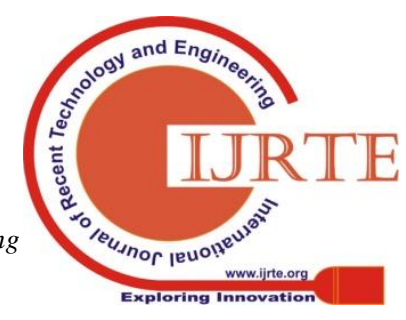

